Bull. Korean Math. Soc. 48 (2011), No. 5, pp. 991-1002

http://dx.doi.org/10.4134/BKMS.2011.48.5.991

\title{
AN ITERATION SCHEMES FOR NONEXPANSIVE MAPPINGS AND VARIATIONAL INEQUALITIES
}

\author{
HongJun Wang And Yisheng Song
}

\begin{abstract}
An iterative algorithm is provided to find a common element of the set of fixed points of a nonexpansive mapping and the set of solutions of some variational inequality in a Hilbert space. Using this result, we consider a strong convergence result for finding a common fixed point of a nonexpansive mapping and a strictly pseudocontractive mapping. Our results include the previous results as special cases and can be viewed as an improvement and refinement of the previously known results.
\end{abstract}

\section{Introduction}

Let $H$ be a real Hilbert space with inner product $\langle\cdot, \cdot\rangle$ and norm $\|\cdot\|$, and $K$ be a closed convex subset of $H$. We write $x_{n} \rightarrow x$ to indicate that the sequence $\left\{x_{n}\right\}$ converges weakly to $x . x_{n} \rightarrow x$ implies that $\left\{x_{n}\right\}$ converges strongly to $x$. Let $A: K \rightarrow H$ be a nonlinear operator. The variational inequality problem is to find a $x^{*} \in K$ such that

$$
\left\langle v-x^{*}, A x^{*}\right\rangle \geq 0 \text { for all } v \in K,
$$

which is known as the variational inequality introduced and studied by Stampacchia [27] in 1964. It has been shown that a wide class of problems arising in several branches of pure and applied sciences can be studied in the unified and general framework of variational inequalities. For example, Noor [12] considered the local and global uniqueness of the solution and sensitivity analysis of the general variational in equalities as well as the finite convergence of the projection-type algorithms; Noor and Bnouhachem [14] analyzed a new three-step iterative algorithm for solving the general variational inequalities and studied its global convergence under some mild conditions; Using the projection technique, Noor and Huang [16] established the equivalence between the Wiener-Hopf equations and variational inequalities; Qin and Noor [18] proved the general variational inequality problems are equivalent to solving the general Wiener-Hopf equations. Other known results see [5, 6, 3, 8, 10, 11, 29, 30, 31]

Received February 11, 2010; Revised May 1, 2010.

2010 Mathematics Subject Classification. 47H06, 47J05, 47J25, 47H10, 47H17.

Key words and phrases. metric projection, inverse-strongly monotone mapping, nonexpansive mapping, variational inequality, strong convergence. 
and the references therein. The set of solutions of the variational inequality is denoted by $V I(K, A)$.

Let $T$ be a mapping with domain $D(T)$ and range $R(T)$ in $E$. $T$ is called nonexpansive if $\|T x-T y\| \leq\|x-y\|$ for all $x, y \in D(T)$. We denote by $F(T)$ the set of fixed points of $T$. Related to the results of such a class of mappings, see $[4,9,19,20,24,22,23,26,25]$ and the references therein.

For every point $x \in H$, there exists a unique nearest point in $K$, denoted by $P_{K} x$, such that $\left\|x-P_{K} x\right\| \leq\|x-y\|$ for all $y \in K$. $P_{K}$ is called the metric projection of $H$ onto $K$. We know that $P_{K}$ is a nonexpansive mapping of $H$ onto $K$. It is also known that $P_{K}$ satisfies

$$
\left\langle x-y, P_{K} x-P_{K} y\right\rangle \geq\left\|P_{K} x-P_{K} y\right\|^{2} \text { for all } x, y \in H .
$$

Moreover, $P_{K} x$ is characterized by the properties:

$$
P_{K} x \in K \text { and }\left\langle x-P_{K} x, P_{K} x-y\right\rangle \geq 0 \text { for all } y \in K \text {. }
$$

In the context of the variational inequality problem, this implies that

$$
x^{*} \in V I(K, A) \text { if and only if } x^{*}=P_{K}\left(x^{*}-\rho A x^{*}\right), \forall \rho>0 .
$$

This means the equivalence between the variational inequalities and fixed point problems using the projection technique. This alternative equivalent formulation has played an important role in developing the some efficient numerical techniques for solving variational inequalities and related optimization problems. Related to the variational inequalities, is the problem of finding the fixed points of the nonexpansive mappings, which is the subject of current interest in functional analysis.

In order to finding the common elements of the set of the solutions of some class of variational inequalities and the set of the fixed points of nonexpansive mappings, Huang and Noor [10] analyzed a class of unified iteration schemes with errors; Noor and Huang [15] considered the convergence criteria of threestep iteration methods; Noor [13] obtained the convergence analysis of some three-step iterative schemes for the Noor variational inequalities involving two nonlinear operators; Qin and Noor [18] established a general iterative algorithm for general variational inequalities and general Wiener-Hopf equations; Qin, Cho and Kang [17] studied strong convergence of an iterative algorithm for a system of generalized variational inequalities; Bnouhachem, Noor and Hao [3] proved the strong convergence of some new extragradient iterative methods for the variational inequality for an inverse strongly monotone mapping in a Hilbert space.

Inspired and motivated by the above research, we suggest and analyze an iterative scheme for finding a common element of the set of fixed points of a nonexpansive mapping and the set of solutions of some variational inequality in a real Hilbert space. Under mild assumptions, we obtain that this iterative sequence converges strongly to a common element of two sets. Using this result, we obtain a strong convergence theorem for finding a common fixed point of a 
nonexpansive mapping and a strictly pseudocontractive mapping. Our results can be viewed as refinement of the previously known results such as Huang and Noor [10] and others.

\section{Preliminaries and basic results}

Let $K$ be a nonempty closed convex subset of a Hilbert space $H$. A mapping $A$ of $K$ into $H$ is called inverse-strongly monotone if there exists a positive real number $\alpha$ such that

$$
\langle x-y, A x-A y\rangle \geq \alpha\|A x-A y\|^{2} \text { for all } x, y \in K
$$

see $[6,11,31]$. For such a case, $A$ is called $\alpha$-inverse-strongly monotone. Clearly, the metric projection of $K$ into $H$ is 1-inverse-strongly monotone. If $A=$ $I-T$, where $T$ is a nonexpansive mapping of $K$ into itself and $I$ is the identity mapping of $H$, then $A$ is $\frac{1}{2}$-inverse-strongly monotone and $V I(K, A)=F(T)$. In fact,

$$
\begin{aligned}
\|A x-A y\|^{2} & =\langle A x-A y, x-y\rangle-\langle x-T x-(y-T y), T x-T y\rangle \\
& =\langle A x-A y, x-y\rangle-\langle x-y, T x-T y\rangle+\|T x-T y\|^{2} \\
& \leq\langle A x-A y, x-y\rangle-\langle x-y, T x-T y\rangle+\langle x-y, x-y\rangle \\
& =2\langle A x-A y, x-y\rangle .
\end{aligned}
$$

Recall a mapping $T: K \rightarrow K$ is said to be strictly pseudocontractive in the sense of Browder and Petryshyn [7] if for any $x, y \in K$ and some $k \in(0,1)$,

$$
\langle T x-T y, x-y\rangle \leq\|x-y\|^{2}-k\|(x-T x)-(y-T y)\|^{2} .
$$

It is easy to see that such mappings are Lipschitz with a Lipschitz constant $L=\frac{1+k}{k}$ and $A$ is $k$-inverse-strongly monotone and $V I(K, A)=F(T)$ whenever $A=I-T$.

If $A$ is an $\alpha$-inverse-strongly monotone mapping of $K$ into $H$, then it is obvious that $A$ is $\frac{1}{\alpha}$-Lipschitz continuous. We also have that for some $\lambda \in$ $(0,2 \alpha], I-\lambda A$ is a nonexpansive mapping of $K$ into $H$. Actually, for all $x, y \in K$,

$$
\begin{aligned}
\|(I-\lambda A) x-(I-\lambda A) y\|^{2} & =\|x-y-\lambda(T x-T y)\|^{2} \\
& =\|x-y\|^{2}-2 \lambda\langle x-y, A x-A y\rangle+\lambda^{2}\|A x-A y\|^{2} \\
& \leq\|x-y\|^{2}+\lambda(\lambda-2 \alpha)\|A x-A y\|^{2} \\
& \leq\|x-y\|^{2} .
\end{aligned}
$$

In order to proving our main results, we also the following.

Lemma 2.1 (T. Suzuki [28, Lemma 2]). Let $\left\{x_{n}\right\}$ and $\left\{y_{n}\right\}$ be two bounded sequences in a Banach space $E$ and $\beta_{n} \in[0,1]$ with $0<\liminf _{n \rightarrow \infty} \beta_{n} \leq$ $\limsup _{n \rightarrow \infty} \beta_{n}<1$. Suppose $x_{n+1}=\beta_{n} x_{n}+\left(1-\beta_{n}\right) y_{n}$ for all integers $n \geq 0$ and $\lim \sup _{n \rightarrow \infty}\left(\left\|y_{n+1}-y_{n}\right\|-\left\|x_{n+1}-x_{n}\right\|\right) \leq 0$. Then $\lim _{n \rightarrow \infty}\left\|x_{n}-y_{n}\right\|=0$. 
Lemma 2.2 (see $[1,2]$ ). Let $\left\{\lambda_{n}\right\}$ and $\left\{\beta_{n}\right\}$ be two sequences of nonnegative real numbers and $\left\{\alpha_{n}\right\}$ a sequence of positive numbers satisfying the conditions $\sum_{n=0}^{\infty} \gamma_{n}=\infty$ and $\lim _{n \rightarrow \infty} \frac{\beta_{n}}{\alpha_{n}}=0$. Let the recursive inequality

$$
\lambda_{n+1} \leq \lambda_{n}-\alpha_{n} \psi\left(\lambda_{n}\right)+\beta_{n}, \quad n=0,1,2, \ldots,
$$

be given where $\psi(\lambda)$ is a continuous and strict increasing function for all $\lambda \geq$ 0 with $\psi(0)=0$. Then $\left\{\lambda_{n}\right\}$ converges to zero, as $n \rightarrow \infty$; there exists $a$ subsequence $\left\{\lambda_{n_{k}}\right\} \subset\left\{\lambda_{n}\right\}, k=1,2, \ldots$, such that

$$
\begin{aligned}
& \lambda_{n_{k}} \leq \psi^{-1}\left(\frac{1}{\sum_{m=0}^{n_{k}} \alpha_{m}}+\frac{\beta_{n_{k}}}{\alpha_{n_{k}}}\right), \\
& \lambda_{n_{k}+1} \leq \psi^{-1}\left(\frac{1}{\sum_{m=0}^{n_{k}} \alpha_{m}}+\frac{\beta_{n_{k}}}{\alpha_{n_{k}}}\right)+\beta_{n_{k}}, \\
& \lambda_{n} \leq \lambda_{n_{k}+1}-\sum_{m=n_{k}+1}^{n-1} \frac{\alpha_{m}}{\theta_{m}}, n_{k}+1<n<n_{k+1}, \theta_{m}=\sum_{i=0}^{m} \alpha_{i}, \\
& \lambda_{n+1} \leq \lambda_{0}-\sum_{m=0}^{n} \frac{\alpha_{m}}{\theta_{m}} \leq \lambda_{0}, 1 \leq n \leq n_{k}-1, \\
& 1 \leq n_{k} \leq s_{\max }=\max \left\{s ; \sum_{m=0}^{s} \frac{\alpha_{m}}{\theta_{m}} \leq \lambda_{0}\right\} .
\end{aligned}
$$

\section{Strong convergent theorems}

Theorem 3.1. Let $K$ be a nonempty closed convex subset of a Hilbert space $H$. Assume that $A: K \rightarrow H$ is an $\alpha$-inverse-strongly monotone mapping and $T: K \rightarrow K$ is a nonexpansive self-mapping with $V I(K, A) \cap F(T) \neq \emptyset$. For an anchor point $u \in K$ and an initial value $x_{0} \in K$ and a constant $\lambda \in(0,2 \alpha)$, the sequence $\left\{x_{n}\right\}$ be defined iteratively by

$$
x_{n+1}=\alpha_{n} u+\beta_{n} x_{n}+\left(1-\alpha_{n}-\beta_{n}\right) T P_{K}\left(x_{n}-\lambda A x_{n}\right) .
$$

Suppose that $\left\{\alpha_{n}\right\} \subset(0,1)$ and $\left\{\beta_{n}\right\} \subset(0,1)$ satisfy the following conditions:

(C1) $\lim _{n \rightarrow \infty} \alpha_{n}=0$; (C2) $\sum_{n=1}^{\infty} \alpha_{n}=\infty$; (B) $0<\liminf _{n \rightarrow \infty} \beta_{n} \leq \limsup _{n \rightarrow \infty} \beta_{n}<1$.

Then $\left\{x_{n}\right\}$ converges strongly to $x^{*}=P_{V I(K, A) \cap F(T)} u$. Moreover, there exist a subsequence $\left\{x_{n_{k}}\right\} \subset\left\{x_{n}\right\}$ and $\left\{\varepsilon_{n}\right\} \subset(0,+\infty)$ with $\lim _{n \rightarrow \infty} \varepsilon_{n}=0$ such that

$$
\begin{aligned}
& \left\|x_{n_{k}}-x^{*}\right\|^{2} \leq \frac{1}{\sum_{m=0}^{n_{k}} \alpha_{m}}+2 \varepsilon_{n_{k}}, \\
& \left\|x_{n_{k}+1}-x^{*}\right\|^{2} \leq \frac{1}{\sum_{m=0}^{n_{k}} \alpha_{m}}+\left(1+2 \alpha_{n_{k}}\right) \varepsilon_{n_{k}}, \\
& \left\|x_{n}-x^{*}\right\|^{2} \leq\left\|x_{n_{k}+1}-P u\right\|^{2}-\sum_{m=n_{k}+1}^{n-1} \frac{\alpha_{m}}{\theta_{m}}, n_{k}+1<n<n_{k+1}, \theta_{m}=\sum_{i=0}^{m} \alpha_{i},
\end{aligned}
$$




$$
\begin{aligned}
& \left\|x_{n+1}-x^{*}\right\|^{2} \leq\left\|x_{0}-x^{*}\right\|^{2}-\sum_{m=0}^{n} \frac{\alpha_{m}}{\theta_{m}} \leq\left\|x_{0}-x^{*}\right\|^{2}, 1 \leq n \leq n_{k}-1, \\
& 1 \leq n_{k} \leq s_{\max }=\max \left\{s ; \sum_{m=0}^{s} \frac{\alpha_{m}}{\theta_{m}} \leq\left\|x_{0}-x^{*}\right\|^{2}\right\} .
\end{aligned}
$$

Proof. Let $z_{n}=P_{K}\left(x_{n}-\lambda A x_{n}\right)$ and $y_{n}=\frac{\alpha_{n}}{1-\beta_{n}} u+\left(1-\frac{\alpha_{n}}{1-\beta_{n}}\right) T z_{n}$. Take $p \in V I(K, A) \cap F(T)$. Then from the nonexpansivity of $I-\lambda A$ and Eq.(1.3), we have that

$$
\begin{aligned}
& \left\|x_{n+1}-p\right\| \\
\leq & \alpha_{n}\|u-p\|+\beta_{n}\left\|x_{n}-p\right\|+\left(1-\alpha_{n}-\beta_{n}\right)\left\|T P_{K}\left(x_{n}-\lambda A x_{n}\right)-p\right\| \\
\leq & \alpha_{n}\|u-p\|+\beta_{n}\left\|x_{n}-p\right\|+\left(1-\alpha_{n}-\beta_{n}\right)\left\|P_{K}\left(x_{n}-\lambda A x_{n}\right)-P_{K}(p-\lambda A p)\right\| \\
\leq & \alpha_{n}\|u-p\|+\beta_{n}\left\|x_{n}-p\right\|+\left(1-\alpha_{n}-\beta_{n}\right)\left\|(I-\lambda A) x_{n}-(I-\lambda A) p\right\| \\
\leq & \alpha_{n}\|u-p\|+\left(1-\alpha_{n}\right)\left\|x_{n}-p\right\| \\
\leq & \max \left\{\left\|x_{n}-p\right\|,\|u-p\|\right\} \\
& \vdots \\
\leq & \max \left\{\left\|x_{0}-p\right\|,\|u-p\|\right\} .
\end{aligned}
$$

So the set $\left\{x_{n}\right\}$ is bounded. This implies the boundedness of the sets $\left\{y_{n}\right\}$ and $\left\{z_{n}\right\}$ since $\left\|z_{n}-p\right\| \leq\left\|P_{K}\left(x_{n}-\lambda A x_{n}\right)-P_{K}(p-\lambda A p)\right\| \leq\left\|x_{n}-p\right\|$ and

$$
\begin{aligned}
\left\|y_{n}-p\right\| & \leq \frac{\alpha_{n}}{1-\beta_{n}}\|u-p\|+\left(1-\frac{\alpha_{n}}{1-\beta_{n}}\right)\left\|T z_{n}-p\right\| \\
& \leq \frac{\alpha_{n}}{1-\beta_{n}}\|u-p\|+\left(1-\frac{\alpha_{n}}{1-\beta_{n}}\right)\left\|z_{n}-p\right\| .
\end{aligned}
$$

Let $M=\sup _{n \in \mathbb{N}}\left\{\|u\|,\left\|x_{n}\right\|,\left\|y_{n}\right\|,\left\|z_{n}\right\|,\left\|x_{n}-p\right\|,\left\|y_{n}-p\right\|\right\}$, where $\mathbb{N}$ denotes all positive integer.

Setting $\gamma_{n}=\frac{\alpha_{n}}{1-\beta_{n}}$. Then by the conditions (C1) and (B), we have $\lim _{n \rightarrow \infty} \gamma_{n}$ $=0$ and $y_{n}=\gamma_{n} u+\left(1-\gamma_{n}\right) T z_{n}$. Further, we also have

$$
\begin{aligned}
& \left\|y_{n+1}-y_{n}\right\| \\
= & \left\|\left(\gamma_{n+1}-\gamma_{n}\right) u+\left(1-\gamma_{n+1}\right) T z_{n+1}-\gamma_{n} T z_{n+1}+\gamma_{n} T z_{n+1}-\left(1-\gamma_{n}\right) T z_{n}\right\| \\
\leq & \left|\gamma_{n+1}-\gamma_{n}\right|\left(\|u\|+\left\|T z_{n+1}\right\|\right)+\left(1-\gamma_{n}\right)\left\|T z_{n+1}-T z_{n}\right\| \\
\leq & 2 M\left|\gamma_{n+1}-\gamma_{n}\right|+\left(1-\gamma_{n}\right)\left\|P_{K}(I-\lambda A) x_{n+1}-P_{K}(I-\lambda A) x_{n}\right\| \\
\leq & 2 M\left|\gamma_{n+1}-\gamma_{n}\right|+\left\|x_{n+1}-x_{n}\right\| .
\end{aligned}
$$

Therefore, $\left\|y_{n+1}-y_{n}\right\|-\left\|x_{n+1}-x_{n}\right\| \leq 2 M\left|\gamma_{n+1}-\gamma_{n}\right| \rightarrow 0$ as $n \rightarrow \infty$. Hence,

$$
\limsup _{n \rightarrow \infty}\left(\left\|y_{n+1}-y_{n}\right\|-\left\|x_{n+1}-x_{n}\right\|\right) \leq 0 \text {. }
$$

By the definition (3.1) of the sequence $\left\{x_{n}\right\}$, we have

$$
x_{n+1}=\beta_{n} x_{n}+\left(1-\beta_{n}\right) y_{n} .
$$


Thus, an application of Lemma 2.1 yields

$$
\lim _{n \rightarrow \infty}\left\|y_{n}-x_{n}\right\|=0 \text {. }
$$

From (3.3) and $\lim _{n \rightarrow \infty} \gamma_{n}=0$, we have

$$
\lim _{n \rightarrow \infty}\left\|y_{n}-T z_{n}\right\|=\lim _{n \rightarrow \infty} \gamma_{n}\left\|u-T z_{n}\right\|=0 .
$$

Combining (3.3) and (3.4) to get

$$
\lim _{n \rightarrow \infty}\left\|x_{n}-T z_{n}\right\|=0 .
$$

We claim that $\lim _{n \rightarrow \infty}\left\|z_{n}-T z_{n}\right\|=0$. From $y_{n}=\gamma_{n} u+\left(1-\gamma_{n}\right) T z_{n}$ and Eq.(2.3), we have

$$
\begin{aligned}
\left\|y_{n}-p\right\|^{2} & \leq \gamma_{n}\|u-p\|^{2}+\left(1-\gamma_{n}\right)\left\|T z_{n}-p\right\|^{2} \\
& \leq \gamma_{n}\|u-p\|^{2}+\left(1-\gamma_{n}\right)\left\|P_{K}\left(x_{n}-\lambda A x_{n}\right)-P_{K}(p-\lambda A p)\right\|^{2} \\
& \leq \gamma_{n}\|u-p\|^{2}+\left(1-\gamma_{n}\right)\left(\left\|x_{n}-p\right\|^{2}+\lambda(\lambda-2 \alpha)\left\|A x_{n}-A p\right\|^{2}\right) .
\end{aligned}
$$

Therefore,

$$
\begin{aligned}
& \left(1-\gamma_{n}\right) \lambda(2 \alpha-\lambda)\left\|A x_{n}-A p\right\|^{2} \\
\leq & \gamma_{n}\|u-p\|^{2}+\left\|x_{n}-p\right\|^{2}-\left\|y_{n}-p\right\|^{2} \\
\leq & \gamma_{n}\|u-p\|^{2}+\left(\left\|x_{n}-p\right\|+\left\|y_{n}-p\right\|\right)\left\|x_{n}-y_{n}\right\| .
\end{aligned}
$$

That is,

$$
\left\|A x_{n}-A p\right\|^{2} \leq \frac{\gamma_{n}\|u-p\|^{2}+2 M\left\|x_{n}-y_{n}\right\|}{\left(1-\gamma_{n}\right) \lambda(2 \alpha-\lambda)} .
$$

On the other hand, noting Eq.(1.1),

$$
\begin{aligned}
\left\|z_{n}-p\right\|^{2}= & \left\|P_{K}\left(x_{n}-\lambda A x_{n}\right)-P_{K}(p-\lambda A p)\right\|^{2} \\
\leq & \left\langle z_{n}-p,\left(x_{n}-\lambda A x_{n}\right)-(p-\lambda A p)\right\rangle \\
= & \frac{1}{2}\left[\left\|\left(x_{n}-\lambda A x_{n}\right)-(p-\lambda A p)\right\|^{2}+\left\|z_{n}-p\right\|^{2}\right. \\
& \left.-\left\|\left(x_{n}-\lambda A x_{n}\right)-(p-\lambda A p)-\left(z_{n}-p\right)\right\|^{2}\right] \\
\leq & \frac{1}{2}\left[\left\|x_{n}-p\right\|^{2}+\left\|z_{n}-p\right\|^{2}-\left\|\left(x_{n}-z_{n}\right)+\lambda\left(A p-A x_{n}\right)\right\|^{2}\right] \\
= & \frac{1}{2}\left[\left\|x_{n}-p\right\|^{2}+\left\|z_{n}-p\right\|^{2}-\left\|x_{n}-z_{n}\right\|^{2}\right. \\
& \left.-2 \lambda\left\langle x_{n}-z_{n}, A p-A x_{n}\right\rangle-\lambda^{2}\left\|A p-A x_{n}\right\|^{2}\right] .
\end{aligned}
$$

Substituting Eq.(3.6) in above equation to get

$$
\begin{aligned}
\left\|z_{n}-p\right\|^{2} & \leq\left\|x_{n}-p\right\|^{2}-\left\|x_{n}-z_{n}\right\|^{2}+2 \lambda\left\langle x_{n}-z_{n}, A x_{n}-A p\right\rangle \\
& \leq\left\|x_{n}-p\right\|^{2}-\left\|x_{n}-z_{n}\right\|^{2}+4 M \lambda \sqrt{\frac{\gamma_{n}\|u-p\|^{2}+2 M\left\|x_{n}-y_{n}\right\|}{\left(1-\gamma_{n}\right) \lambda(2 \alpha-\lambda)}} .
\end{aligned}
$$


Thus,

$$
\begin{aligned}
\left\|y_{n}-p\right\|^{2} \leq & \gamma_{n}\|u-p\|^{2}+\left(1-\gamma_{n}\right)\left\|z_{n}-p\right\|^{2} \\
\leq & \gamma_{n}\|u-p\|^{2}+\left(1-\gamma_{n}\right)\left\|x_{n}-p\right\|^{2} \\
& \quad-\left(1-\gamma_{n}\right)\left\|x_{n}-z_{n}\right\|^{2}+4 M \lambda \sqrt{\frac{\gamma_{n}\|u-p\|^{2}+2 M\left\|x_{n}-y_{n}\right\|}{\left(1-\gamma_{n}\right) \lambda(2 \alpha-\lambda)}} .
\end{aligned}
$$

Moreover,

$$
\begin{aligned}
\left(1-\gamma_{n}\right)\left\|x_{n}-z_{n}\right\|^{2} \leq & \gamma_{n}\|u-p\|^{2}+\left\|x_{n}-p\right\|^{2}-\left\|y_{n}-p\right\|^{2} \\
& +4 M \lambda \sqrt{\frac{\gamma_{n}\|u-p\|^{2}+2 M\left\|x_{n}-y_{n}\right\|}{\left(1-\gamma_{n}\right) \lambda(2 \alpha-\lambda)}} \\
\leq & \gamma_{n}\|u-p\|^{2}+2 M\left\|x_{n}-y_{n}\right\| \\
& +4 M \lambda \sqrt{\frac{\gamma_{n}\|u-p\|^{2}+2 M\left\|x_{n}-y_{n}\right\|}{\left(1-\gamma_{n}\right) \lambda(2 \alpha-\lambda)}}
\end{aligned}
$$

Since $\gamma_{n} \rightarrow 0$ and $\left\|x_{n}-y_{n}\right\| \rightarrow 0$, we obtain

$$
\lim _{n \rightarrow \infty}\left\|x_{n}-z_{n}\right\|=0
$$

Combining Eq.(3.5) to get

$$
\lim _{n \rightarrow \infty}\left\|z_{n}-T z_{n}\right\|=0 .
$$

Next we show that for $x^{*}=P_{V I(K, A) \cap F(T)} u$,

$$
\limsup _{n \rightarrow \infty}\left\langle u-x^{*}, x_{n+1}-x^{*}\right\rangle \leq 0 .
$$

To show it, choose a subsequence $\left\{x_{n_{k}}\right\}$ of $\left\{x_{n}\right\}$ such that

$$
\limsup _{n \rightarrow \infty}\left\langle u-x^{*}, x_{n}-x^{*}\right\rangle=\lim _{k \rightarrow \infty}\left\langle u-x^{*}, x_{n_{k}}-x^{*}\right\rangle \text {. }
$$

We may assume that $x_{n_{k}} \rightarrow q$ as $\left\{x_{n}\right\}$ is bounded. Since $\lim _{n \rightarrow \infty}\left\|x_{n}-z_{n}\right\|=$ 0 , this means $z_{n_{k}} \rightarrow q$. Then we can obtain $q \in V I(K, A) \cap F(T)$. In fact, since

$$
\begin{aligned}
& \left\|z_{n_{k}}-q\right\|^{2}+2\left\langle z_{n_{k}}-q, q-T q\right\rangle+\|q-T q\|^{2} \\
= & \left\|z_{n_{k}}-T q\right\|^{2} \\
\leq & \left(\left\|z_{n_{k}}-T z_{n_{k}}\right\|+\left\|T z_{n_{k}}-T q\right\|\right)^{2} \\
\leq & \left(\left\|z_{n_{k}}-T z_{n_{k}}\right\|+\left\|z_{n_{k}}-q\right\|\right)^{2} .
\end{aligned}
$$

Consequently,

$$
2\left\langle z_{n_{k}}-q, q-T q\right\rangle+\|q-T q\|^{2} \leq\left\|z_{n_{k}}-T z_{n_{k}}\right\|\left(\left\|z_{n_{k}}-T z_{n_{k}}\right\|+2\left\|z_{n_{k}}-q\right\|\right) .
$$

Let $k \rightarrow \infty$, noting Eq.(3.8) and $z_{n_{k}} \rightarrow q$, we obtain $q \in F(T)$. Similarly, since the mapping $P_{K}(I-\lambda A)$ is nonexpansive and

$$
\lim _{n \rightarrow \infty}\left\|x_{n}-z_{n}\right\|=\lim _{n \rightarrow \infty}\left\|x_{n}-P_{K}(I-\lambda A) x_{n}\right\|=0
$$


we also obtain that $q=P_{K}(I-\lambda A) q$. Applying Eq.(1.3) to yield $q \in V I(K, A)$.

Hence, it follows from Eq.(1.2) and $x_{n_{k}} \rightarrow q$ that

$\limsup _{n \rightarrow \infty}\left\langle u-x^{*}, x_{n}-x^{*}\right\rangle=\lim _{k \rightarrow \infty}\left\langle u-x^{*}, x_{n_{k}}-x^{*}\right\rangle=\left\langle u-x^{*}, q-x^{*}\right\rangle \leq 0$.

Put $\varepsilon_{n}=\max \left\{\left\langle u-x^{*}, x_{n+1}-x^{*}\right\rangle, 0\right\}$. Then it is obvious that $\left\{\varepsilon_{n}\right\} \subset(0,+\infty)$ with $\lim _{n \rightarrow \infty} \varepsilon_{n}=0$ and $\left\langle u-x^{*}, x_{n+1}-x^{*}\right\rangle \leq \varepsilon_{n}$.

Finally, we show that $x_{n} \rightarrow x^{*}$. In fact, since

$$
\left\|z_{n}-x^{*}\right\| \leq\left\|P_{K}\left(x_{n}-\lambda A x_{n}\right)-P_{K}\left(x^{*}-\lambda A x^{*}\right)\right\| \leq\left\|x_{n}-x^{*}\right\|,
$$

then

$$
\begin{aligned}
& \left\|x_{n+1}-x^{*}\right\|^{2} \\
= & \left\langle\beta_{n}\left(x_{n}-x^{*}\right)+\left(1-\alpha_{n}-\beta_{n}\right)\left(T z_{n}-x^{*}\right)+\alpha_{n}\left(u-x^{*}\right), x_{n+1}-x^{*}\right\rangle \\
\leq & \left(\beta_{n}\left\|x_{n}-x^{*}\right\|+\left(1-\alpha_{n}-\beta_{n}\right)\left\|T z_{n}-x^{*}\right\|\right)\left\|x_{n+1}-x^{*}\right\| \\
& +\alpha_{n}\left\langle u-x^{*}, x_{n+1}-x^{*}\right\rangle \\
\leq & \left(1-\alpha_{n}\right)\left\|x_{n}-x^{*}\right\|\left\|x_{n+1}-x^{*}\right\|+\alpha_{n} \varepsilon_{n} \\
\leq & \left(1-\alpha_{n}\right) \frac{\left\|x_{n}-x^{*}\right\|^{2}+\left\|x_{n+1}-x^{*}\right\|^{2}}{2}+\alpha_{n} \varepsilon_{n} .
\end{aligned}
$$

Namely,

$$
\left\|x_{n+1}-x^{*}\right\|^{2} \leq\left(1-\alpha_{n}\right)\left\|x_{n}-x^{*}\right\|^{2}+2 \alpha_{n} \varepsilon_{n} .
$$

Hence, an application of Lemma $2.2(\psi(t)=t)$ yields that $\left\{x_{n}\right\}$ strongly converges to $x^{*}$. And the remainder estimates now follow from Lemma 2.2.

Corollary 3.2. Let $H, T, A, K, \lambda$ be as Theorem 3.1. For an anchor point $u \in K$ and an initial value $x_{0} \in K$ and a constant $\delta \in(0,1)$, the sequence $\left\{x_{n}\right\}$ be defined iteratively by

$$
x_{n+1}=\alpha_{n} u+\left(1-\alpha_{n}\right)\left[\delta x_{n}+(1-\delta) T P_{K}\left(x_{n}-\lambda A x_{n}\right)\right] .
$$

Suppose that $\left\{\alpha_{n}\right\} \subset(0,1)$ satisfies $(\mathrm{C} 1) \lim _{n \rightarrow \infty} \alpha_{n}=0 ;(\mathrm{C} 2) \sum_{n=1}^{\infty} \alpha_{n}=\infty$. Then $\left\{x_{n}\right\}$ converges strongly to $P_{V I(K, A) \cap F(T)} u$.

Proof. By the definition (3.11) of the sequence $\left\{x_{n}\right\}$, we have that

$$
x_{n+1}=\alpha_{n} u+\left(1-\alpha_{n}\right) \delta x_{n}+(1-\delta)\left(1-\alpha_{n}\right) T P_{K}\left(x_{n}-\lambda A x_{n}\right) .
$$

Then

$$
\alpha_{n}+\left(1-\alpha_{n}\right) \delta+(1-\delta)\left(1-\alpha_{n}\right)=1 \quad \text { and } \quad 0<\lim _{n \rightarrow \infty}\left(1-\alpha_{n}\right) \delta=\delta<1 .
$$

Proceeding as in Theorem 3.1, we reach the conclusion.

Corollary 3.3. Let $H, T, A, K, \lambda$ be as Theorem 3.1. For an anchor point $u \in K$ and an initial value $x_{0} \in K$ and a constant $\delta \in(0,1)$, the sequence $\left\{x_{n}\right\}$ be defined iteratively by

$$
x_{n+1}=\delta\left(\alpha_{n} u+\left(1-\alpha_{n}\right) x_{n}\right)+(1-\delta) T P_{K}\left(x_{n}-\lambda A x_{n}\right) .
$$


Suppose that $\left\{\alpha_{n}\right\} \subset(0,1)$ satisfies $(\mathrm{C} 1) \lim _{n \rightarrow \infty} \alpha_{n}=0 ;(\mathrm{C} 2) \sum_{n=1}^{\infty} \alpha_{n}=\infty$. Then $\left\{x_{n}\right\}$ converges strongly to $P_{V I(K, A) \cap F(T)} u$.

Proof. By the definition (3.12) of the sequence $\left\{x_{n}\right\}$, we have that

$$
x_{n+1}=\delta \alpha_{n} u+\left(1-\alpha_{n}\right) \delta x_{n}+(1-\delta) T P_{K}\left(x_{n}-\lambda A x_{n}\right) .
$$

Then $\delta \alpha_{n}$ satisfies the conditions (C1) and (C2),

$$
\delta \alpha_{n}+\left(1-\alpha_{n}\right) \delta+(1-\delta)=1 \quad \text { and } \quad 0<\lim _{n \rightarrow \infty}\left(1-\alpha_{n}\right) \delta=\delta<1
$$

Proceeding as in Theorem 3.1, we reach the conclusion.

Corollary 3.4. Let $K, H,\left\{\alpha_{n}\right\},\left\{\beta_{n}\right\}$ be as Theorem 3.1. Assumed that $A$ : $K \rightarrow H$ is an $\alpha$-inverse-strongly monotone mapping with $\operatorname{VI}(K, A) \neq \emptyset$. For an anchor point $u \in K$ and an initial value $x_{0} \in K$ and a constant $\lambda \in(0,2 \alpha)$, the sequence $\left\{x_{n}\right\}$ be defined iteratively by

$$
x_{n+1}=\alpha_{n} u+\beta_{n} x_{n}+\left(1-\alpha_{n}-\beta_{n}\right) P_{K}\left(x_{n}-\lambda A x_{n}\right) .
$$

Then $\left\{x_{n}\right\}$ converges strongly to $P_{V I(K, A)} u$.

\section{Some applications}

In this section, we prove several strong convergence theorems by using Theorem 3.1.

Recall a mapping $f: K \rightarrow K$ is called to be weakly contractive if

$$
\|f(x)-f(y)\| \leq\|x-y\|-\varphi(\|x-y\|) \text { for all } x, y \in K,
$$

for some $\varphi:[0,+\infty) \rightarrow[0,+\infty)$ is a continuous and strictly increasing function such that $\varphi$ is positive on $(0,+\infty)$ and $\varphi(0)=0$. Clearly, the mapping contains contractive mapping as a special case $(\varphi(t)=(1-\beta) t$ for $\beta \in(0,1))$.

Rhoades [21] obtained the result-like Banach's Contraction Mapping Principle for the weakly contractive mapping.

Theorem R ([21, Theorem 2]). Let $(X, d)$ be a complete metric space, and $f$ a weakly contractive mapping on $X$. Then $f$ has a unique fixed point $p$ in $X$. Moreover, for $x \in X,\left\{f^{n}(x)\right\}$ strongly converges to $p$.

Theorem 4.1. Let $K, A, T,\left\{\alpha_{n}\right\},\left\{\beta_{n}\right\}$ be as Theorem 3.1. Assume that $f$ : $K \rightarrow K$ is a weakly contractive mapping with a function $\varphi$. For an initial value $x_{0} \in K$ and a constant $\lambda \in(0,2 \alpha)$, the sequence $\left\{x_{n}\right\}$ be defined iteratively by

$$
x_{n+1}=\alpha_{n} f\left(x_{n}\right)+\beta_{n} x_{n}+\left(1-\alpha_{n}-\beta_{n}\right) T P_{K}\left(x_{n}-\lambda A x_{n}\right) .
$$

Then $\left\{x_{n}\right\}$ converges strongly to $z=P_{V I(K, A) \cap F(T)} f(z)$.

Proof. For any $x, y \in K$, we have

$$
\begin{aligned}
& \left\|P_{V I(K, A) \cap F(T)}(f(x))-P_{V I(K, A) \cap F(T)}(f(y))\right\| \\
\leq & \|f(x)-f(y)\| \leq\|x-y\|-\varphi(\|x-y\|) .
\end{aligned}
$$


So, $P_{V I(K, A) \cap F(T)} f$ is a weakly contractive mapping with a function $\varphi$. Then by Theorem $\mathrm{R}$, there exists a unique element $z \in K$ such that

$$
z=P_{V I(K, A) \cap F(T)}(f(z)) .
$$

Thus we may define a sequence $\left\{y_{n}\right\}$ in $K$ by

$$
y_{n+1}=\alpha_{n} f(z)+\beta_{n} y_{n}+\left(1-\alpha_{n}-\beta_{n}\right) T P_{K}\left(y_{n}-\lambda A y_{n}\right), n=0,1,2, \ldots
$$

Then Theorem 3.1 assures $y_{n} \rightarrow P_{V I(K, A) \cap F(T)}(f(z))=z$ as $n \rightarrow \infty$. For every $n$, we have

$$
\begin{aligned}
\left\|x_{n+1}-y_{n+1}\right\| \leq & \alpha_{n}\left\|f\left(x_{n}\right)-f(z)\right\|+\beta_{n}\left\|x_{n}-y_{n}\right\| \\
& +\left(1-\alpha_{n}-\beta_{n}\right)\left\|T P_{K}\left(x_{n}-\lambda A x_{n}\right)-T P_{K}\left(y_{n}-\lambda A y_{n}\right)\right\| \\
\leq & \alpha_{n}\left(\left\|f\left(x_{n}\right)-f\left(y_{n}\right)\right\|+\left\|f\left(y_{n}\right)-f(z)\right\|\right)+\left(1-\alpha_{n}\right)\left\|x_{n}-y_{n}\right\| \\
\leq & \left\|x_{n}-y_{n}\right\|-\alpha_{n} \varphi\left(\left\|x_{n}-y_{n}\right\|\right)+\alpha_{n}\left(\left\|y_{n}-z\right\|-\varphi\left(\left\|y_{n}-z\right\|\right)\right) \\
\leq & \left\|x_{n}-y_{n}\right\|-\alpha_{n} \varphi\left(\left\|x_{n}-y_{n}\right\|\right)+\alpha_{n}\left\|y_{n}-z\right\| .
\end{aligned}
$$

Since $\left\|y_{n}-z\right\| \rightarrow 0$, then it follows from Lemma 2.2 that $\lim _{n \rightarrow \infty}\left\|x_{n}-y_{n}\right\|=0$. Hence,

$$
\lim _{n \rightarrow \infty}\left\|x_{n}-z\right\| \leq \lim _{n \rightarrow \infty}\left(\left\|x_{n}-y_{n}\right\|+\left\|y_{n}-z\right\|\right)=0 .
$$

Consequently, we obtain the strong convergence of $\left\{x_{n}\right\}$ to

$$
z=P_{V I(K, A) \cap F(T)} f(z) .
$$

This completes the proof.

Theorem 4.2. Let $K, T,\left\{\alpha_{n}\right\},\left\{\beta_{n}\right\}$ be as Theorem 3.1. Assume that $S$ : $K \rightarrow K$ is a strictly pseudocontractive mapping in the sense of Browder and Petryshyn with a constant $k \in(0,1)$ and $f: K \rightarrow K$ is a weakly contractive mapping with a function $\varphi$. For an initial value $x_{0} \in K$ and a constant $\lambda \in$ $(0,2 k)$, the sequence $\left\{x_{n}\right\}$ be defined iteratively by

$$
x_{n+1}=\alpha_{n} f\left(x_{n}\right)+\beta_{n} x_{n}+\left(1-\alpha_{n}-\beta_{n}\right) T\left((1-\lambda) x_{n}+\lambda S x_{n}\right) .
$$

Then $\left\{x_{n}\right\}$ converges strongly to $z=P_{F(S) \cap F(T)} f(z)$.

Proof. Put $A=I-S$. Then $A$ is $k$-inverse-strongly monotone. We have $F(S)=V I(K, A)$ and $P_{K}\left(x_{n}-\lambda A x_{n}\right)=(1-\lambda) x_{n}+\lambda S x_{n}$. So, by Theorem 4.1, we obtain the desired result.

As a direct application of Theorem 3.1, we also have the following.

Theorem 4.3. Let $K, T,\left\{\alpha_{n}\right\},\left\{\beta_{n}\right\}$ be as Theorem 3.1. Assume that $S$ : $K \rightarrow K$ is a strictly pseudocontractive mapping in the sense of Browder and Petryshyn with a constant $k \in(0,1)$. For an anchor point $u \in K$ and an initial value $x_{0} \in K$ and a constant $\lambda \in(0,2 k)$, the sequence $\left\{x_{n}\right\}$ be defined iteratively by

$$
x_{n+1}=\alpha_{n} u+\beta_{n} x_{n}+\left(1-\alpha_{n}-\beta_{n}\right) T\left((1-\lambda) x_{n}+\lambda S x_{n}\right) .
$$

Then $\left\{x_{n}\right\}$ converges strongly to $z=P_{F(S) \cap F(T)} u$. 
Remark. Our results can be viewed as refinement of Huang and Noor [10]. In particular, when in Theorem 4.1, $f(x)=(1-k) x$, Theorem 2.1 of Huang and Noor [10] is reached.

Acknowledgments. The authors are grateful to editors and anonymous referee for their careful reading and valuable comments and suggestions which led to the present form of the paper.

\section{References}

[1] Ya. I. Alber and A. N. Iusem, Extension of subgradient techniques for nonsmooth optimization in Banach spaces, Set-valued Anal. 9 (2001), no. 4, 315-335.

[2] Y. Alber, S. Reich, and J. C. Yao, Iterative methods for solving fixed-point problems with nonself-mappings in Banach spaces, Abstr. Appl. Anal. 2003 (2003), no. 4, 193-216.

[3] A. Bnouhachem, M. A. Noor, and Z. Hao, Some new extragradient iterative methods for variational inequalities, Nonlinear Anal. 70 (2009), no. 3, 1321-1329.

[4] F. E. Browder, Fixed point theorems for noncompact mappings in Hilbert space, Proc. Nat. Acad. Sci. U.S.A. 53 (1965), 1272-1276.

[5] Nonlinear monotone operators and convex sets in Banach spaces, Bull. Amer. Math. Soc. 71 (1965), 780-785.

[6] _ The fixed point theory of multi-valued mappings in topological vector spaces, Math. Ann. 177 (1968), 283-301.

[7] F. E. Browder and W. E. Petryshyn, Construction of fixed points of nonlinear mappings in Hilbert space, J. Math. Anal. Appl. 20 (1967), 197-228.

[8] R. E. Bruck, On the weak convergence of an ergodic iteration for the solution of variational inequalities for monotone operators in Hilbert space, J. Math. Anal. Appl. 61 (1977), no. 1, 159-164.

[9] B. Halpen, Fixed points of nonexpansive maps, Bull. Amer. Math. Soc. 73 (1967), 957961.

[10] Z. Huang and M. A. Noor, Some new unified iteration schemes with errors for nonexpansive mappings and variational inequalities, Appl. Math. Comput. 194 (2007), no. 1, $135-142$.

[11] H. Iiduka and W. Takahashi, Strong convergence theorems for nonexpansive mappings and inverse-strongly monotone mappings, Nonlinear Anal. 61 (2005), no. 3, 341-350.

[12] M. A. Noor, Some developments in general variational inequalities, Appl. Math. Comput. 152 (2004), no. 1, 199-277.

[13] _ General variational inequalities and nonexpansive mappings, J. Math. Anal. Appl. 331 (2007), no. 2, 810-822.

[14] M. A. Noor and A. Bnouhachem, On an iterative algorithm for general variational inequalities, Appl. Math. Comput. 185 (2007), no. 1, 155-168.

[15] M. A. Noor and Z. Huang, Three-step methods for nonexpansive mappings and variational inequalities, Appl. Math. Comput. 187 (2007), no. 2, 680-685.

[16] W Wiener-Hopf equation technique for variational inequalities and nonexpansive mappings, Appl. Math. Comput. 191 (2007), no. 2, 504-510.

[17] X. Qin, S. Y. Cho, and S. M. Kang, Convergence of an iterative algorithm for systems of variational inequalities and nonexpansive mappings with applications, J. Comput. Appl. Math. 233 (2009), no. 2, 231-240.

[18] X. Qin and M. A. Noor, General Wiener-Hopf equation technique for nonexpansive mappings and general variational inequalities in Hilbert spaces, Appl. Math. Comput. 201 (2008), no. 1-2, 716-722.

[19] S. Reich, Strong convergence theorems for resolvents of accretive operators in Banach spaces, J. Math. Anal Appl. 75 (1980), no. 1, 287-292. 
[20] _ Approximating zeros of accretive operators, Proc. Amer. Math. Soc. 51 (1975), no. $2,381-384$

[21] B. E. Rhoades, Some theorems on weakly contractive maps, Nonlinear Anal. 47 (2001), no. 4, 2683-2693.

[22] Y. Song and R. Chen, Convergence theorems of iterative algorithms for continuous pseudocontractive mappings, Nonlinear Anal. 67 (2007), no. 2, 486-497.

[23] Strong convergence theorems on an iterative method for a family of finite nonexpansive mappings, Appl. Math. Comput. 180 (2006), no. 1, 275-287.

[24] _ Viscosity approximation methods for nonexpansive nonself-mappings, J. Math. Anal. Appl. 321 (2006), no. 1, 316-326.

[25] _ Iterative approximation to common fixed points of nonexpansive mapping sequences in reflexive Banach spaces, Nonlinear Anal. 66 (2007), no. 3, 591-603.

[26] Y. Song, R. Chen, and H. Zhou, Viscosity approximation methods for nonexpansive mapping sequences in Banach spaces, Nonlinear Anal. 66 (2007), no. 5, 1016-1024.

[27] G. Stampacchia, Formes bilinéaires coercitives sur les ensembles convexes, C. R. Acad. Sci. Paris 258 (1964), 4413-4416.

[28] T. Suzuki, Strong convergence of Krasnoselskii and Mann's type sequences for oneparameter nonexpansive semigroups without Bochner integrals, J. Math. Anal. Appl. 305 (2005), no. 1, 227-239.

[29] W. Takahashi, Nonlinear variational inequalities and fixed point theorems, J. Math. Soc. Japan 28 (1976), no. 1, 168-181.

[30] _ Nonlinear complementarity problem and systems of convex inequalities, J. Optim. Theory Appl. 24 (1978), no. 3, 499-506.

[31] W. Takahashi and M. Toyoda, Weak convergence theorems for nonexpansive mappings and monotone mappings, J. Optim. Theory Appl. 118 (2003), no. 2, 417-428.

HONGJUN WANG

College of Mathematics and Information Science

Henan Normal University

Henan 453007, P. R. China

E-mail address: wanghj@htu.cn

YISHENG SONG

College of Mathematics and Information Science

Henan Normal University

Henan 453007, P. R. China

E-mail address: songyisheng123@yahoo.com.cn 